\title{
Análise do desempenho reprodutivo e do uso de energia elétrica em instalações climatizadas de cachaços
}

\author{
Luiz A. Rossi ${ }^{1}$, Nina M. Velloso ${ }^{2}$, Marcelo T. de Lima ${ }^{3}$, \\ Juliana Sarubbi ${ }^{4} \&$ Luciano H. S. Vieira ${ }^{5}$
}

\begin{abstract}
RESU M O
O bjetivou-se, com este estudo, avaliar e comparar dois sistemas de climatização do ambiente com dezoito machos da linhagem genética comercial AG337, provenientes da empresa AGRO CERES-PIC. Os animais do grupo $\mathrm{A}$ foram submetidos à climatização por meio de ventilação artificial e nebulização com acionamento automatizado enquanto os do grupo B foram submetidos apenas a ventilação, sendo o acionamento dos equipamentos feito de forma manual e de acordo com o manejo já praticado. Foram considerados aspectos de desempenho reprodutivo e conforto térmico dos animais, além das variáveis climáticas mensuradas no ambiente externo ao galpão e o uso de energia elétrica pelos equipamentos de climatização. Os principais resultados demonstraram que os animais do grupo A permaneceram em condição ambiental mais segura, porém não houve melhora nos índices de qualidade seminal dos animais deste grupo. Ressalta-se que o incremento de custo relativo ao aumento no consumo de energia elétrica, ocasionado pelo uso dos equipamentos de climatização e automação, não acarretará acréscimo significativo na conta de energia pois se trata de equipamentos de baixa demanda de energia elétrica.
\end{abstract}

Palavras-chave: suínos, qualidade seminal, conforto térmico, construções rurais, potência elétrica

\section{Analysis of reproductive performance and use of electrical energy in the acclimatized facilities of boars}

\begin{abstract}
This study aimed to assess and compare two air conditioning systems in a setting where eighteen males of the commercial genetic line AG337, from AGROCERES - PIC, are housed. The animals in G roup A were submitted to automated air conditioning through artificial ventilation and nebulization, while the animals in G roup B were submitted only to manual triggering of the ventilation, according to the schedule practiced at the farm. The factors such as reproductive performance, animal thermal comfort, as well as climatic variables from the external environment of the housing and use of electrical energy by the environment controlling system were considered. The results showed that animals in Group A remained in a comfortable environmental conditions though there was no improvement in the mean values of seminal volume and sperm concentration. It is worthwhile to mention that monthly increase in energy costs due to use of acclimatization and automation equipments were not significant as the equipments are of low energy consumption.
\end{abstract}

Key words: swine, sperm quality, thermal comfort, rural buildings, electric power

\footnotetext{
1 FEAG RI/U NICAM P, Av. Cândido Rondom, 501, Cidade U niversitária Zeferino Vaz, s/n. CEP 13083-875, Campinas, SP. Fone: (19) $3521-1041$. E-mail: rossi@agr.unicamp.br

${ }^{2}$ Rua Diana, 331, Ap. 46, Bairro de Perdizes. CEP 05019-000, São Paulo, SP. Fone: (11) 8156-3636. E-mail: ninamiglioranza@yahoo.com.br ${ }^{3} \mathrm{~N}$ EPP - U niversidade Estadual de Campinas, Avenida Albert Einstein, 1.300, Cidade U niversitária Zeferino Vaz, s/n. CEP 13083-875, Campinas, SP. Fone: (19) 9442-2319. Emails: mtlamazonas@yahoo.com.br; marcelo@nepp.unicamp.br

${ }^{4}$ U FSM , CESN O RS/PM, Av. Independência, 3751, Bairro Vista Alegre. CEP 98300-000, Palmeira das Missões, RS. Fone: (55) 3742-8870. E-mail: jusarubbi.ufsm@hotmail.com

${ }^{5}$ D outorando em Engenharia Agrícola da FEAG RI/U NICAM P, Av. Cândido Rondon, 501, Cidade U niversitária Zeferino Vaz s/n. CEP 13083-875, Campinas, SP. Fone: (19) 8144-8132. Email: vieira.Ihs@gmail.com
} 


\section{INTRODUÇÃO}

O suíno é um animal com aparelho termorregulador pouco desenvolvido (Cavalcanti, 1998; Pedersen, 2002) e, portanto, fatores ambientais externos e o microclima dentro das instalações exercem efeitos diretos e indiretos, em todas as fases de produção.

Para a zona de termoneutralidade de suíno macho reprodutor, considera-se que a faixa de temperatura deve estar entre $18 \mathrm{e}$ $22^{\circ} \mathrm{C}$ (Curtis, 1983), a entalpia entre 60,44 e 68,62 $\mathrm{kJ} \mathrm{kg}^{-1}$ de ar seco e a umidade relativa entre 50 e $70 \%$ (Tolon et al., 2010).

Em locais onde as temperaturas são elevadas, o controle ambiental geralmente é feito por meio de ventilação natural ou artificial e resfriamento evaporativo, o qual se tem mostrado capaz de reduzir as temperaturas no interior das instalações e minimizar o estresse calórico sofrido pelos animais (Bortolozzo et al., 2003; Huynh et al., 2004).

Cachaços submetidos a altas temperaturas ambientais têm sua fertilidade reduzida pois, de acordo com Suriyasomboon (2005), tais condições podem causar uma degeneração testicular leve a moderada, além de desequilíbrio hormonal e metabólico. Esses machos produzem ejaculados com menor concentração de espermatozóides, alta porcentagem de células espermáticas anormais e diminuição da motilidade e do volume espermático.

O tempo mínimo de exposição em relação à temperatura crítica do ar de forma a afetar a produção de células espermáticas, é de respectivamente $72 \mathrm{~h} \mathrm{e} 29,4^{\circ} \mathrm{C}$, sendo um período aproximado de duas semanas, normalmente observado entre o estresse calórico agudo e as primeiras indicações de produção de espermatozóides anormais (Estienne, 2000). Curtis (1983) e Estienne (2000) concluíram que a fertilidade dos cachaços permanece afetada pelas altas temperaturas, por cerca de cinco semanas após o encerramento da exposição dos animais às condições adversas.

De acordo com Suriyasomboon et al. (2004), o volume e a produção espermática total diminuem quando a temperatura e a umidade relativa do ar excedem $30^{\circ} \mathrm{Ce} 40 \%$, respectivamente, no sistema convencional, e $27^{\circ} \mathrm{C} \mathrm{e} 70 \%$, no sistema climatizado.

Uma maneira eficiente de se avaliar os efeitos da temperatura ambiente e da umidade relativa do ar sobre os animais, é mensurar índices de conforto térmico; para isto, pode-se utilizar o índice de temperatura e umidade (ITU) em que valores entre 71 e 78 são considerados críticos; entre 79 e 83 a situação é de perigo e, acima de 83 , é considerado um ambiente em condição de emergência (Silva, 2000). Dentre os parâmetros comumente empregados na avaliação do conforto térmico, a entalpia, energia de ar úmido por unidade de massa de ar seco, é a propriedade mais útil na quantificação de processos psicrométricos que envolvem trocas térmicas (Albright, 1990).

A energia elétrica utilizada nos galpões para alimentação, iluminação e, principalmente, manutenção do conforto térmico animal, tem extrema importância dentro de uma quantificação dos custos de produção e sua relação custo/benefício, no que diz respeito ao consumo de energia elétrica (Bueno \& Rossi, 2006).

A utilização de termostatos, assim como de outros equipamentos indicadores dos índices climáticos, representa um meio significativo de racionalizar o uso da energia elétrica empregada na manutenção da zona de conforto térmico dos animais, podendo gerar economia de até $50 \%$ em relação ao sistema sem sua utilização (Sarubbi et al., 2010).

Sendo assim, o presente trabalho foi desenvolvido com o objetivo de avaliar a eficiência de instalações suinícolas para cachaços sob os aspectos de climatização, considerando-se o desempenho reprodutivo e o conforto térmico dos animais, além do consumo de energia elétrica dos equipamentos de climatização e automação. Para tanto, avaliaram-se a qualidade seminal dos cachaços, a eficácia no uso de ventilação artificial e nebulização automatizadas; por fim, procedeu-se à medição dos parâmetros referentes ao uso de energia elétrica pelos equipamentos.

\section{Material e MÉTODOS}

O trabalho experimental foi realizado em uma granja comercial de suínos localizada no município de Capivari, SP (coordenadas geográficas 22 $2^{\circ} 59^{\prime} 42^{\prime \prime} \mathrm{S}, 47^{\circ} 30^{\prime} 28^{\prime \prime} \mathrm{W}$ e $636 \mathrm{~m}$ ) entre os meses de outubro de 2009 e janeiro de 2010, totalizando 77 dias. O clima da região, segundo a classificação climática de Köppen, é o Cwa, caracterizado pelo clima tropical de altitude, com chuvas no verão e seca no inverno, com a temperatura média do mês mais quente superior a $22{ }^{\circ} \mathrm{C}$. A precipitação pluviométrica média do período registrado foi de $223 \mathrm{~mm}$ ao mês, sendo dezembro de 2009 (319 mm) e janeiro de 2010 (213 $\mathrm{mm})$, respectivamente, os meses de maior e menor índice de precipitação acumulada.

A criação é feita de forma intensiva confinada, ciclo completo e organização semanal da produção, possuindo cerca de 3000 matrizes e 28 cachaços, dos quais 18 (64,3\% da amostra), com idades entre 1 e 3 anos, foram empregados nesta pesquisa por serem da mesma linhagem (AGROCERES - PIC AG 337). Para comprovação do estado de saúde e desempenho reprodutivo, realizou-se uma análise prévia do estado físico e da qualidade seminal.

Os machos foram alojados em baias individuais em um mesmo galpão, sendo as nove primeiras baias ocupadas pelos machos do grupo A e as nove baias posteriores pelos animais do grupo B. A última baia do grupo A e a primeira do grupo B foram separadas por placas de madeira com cobertura plástica, para que a climatização não atingisse o ambiente do grupo controle. A escolha dos animais pertencentes ao grupo A ou B, se deu de maneira a formar dois grupos homogêneos evitandose grande diferença de idade e de desempenho reprodutivo entre os animais, o que poderia comprometer os resultados.

Os animais do grupo A foram submetidos a um sistema automatizado de climatização, no qual se utilizou um controlador automático tipo Humitech II, fabricado pela Full Gauge. Tal controlador recebia dados dos transmissores de temperatura e umidade e, a partir desses dados e de ajustes predefinidos, acionava a ventilação artificial e nebulização. Os ventiladores foram programados para acionar quando a temperatura ambiente do galpão atingisse $25^{\circ} \mathrm{C}$ e desligá-los aos $24^{\circ} \mathrm{C}$. Atingidos 27 ${ }^{\circ} \mathrm{C}$ era acionado o sistema de nebulização, abastecido por uma bomba de $1 \mathrm{cv}$ com pressão de $40 \mathrm{mca}$, programado para desligar quando a temperatura retornasse aos $25^{\circ} \mathrm{C}$ ou quando a umidade 
relativa do ar alcançasse $70 \%$. Os bicos de nebulização possuíam vazão de água de $7 \mathrm{~L} \mathrm{~h}^{-1}$ e dispostos a cada $1,5 \mathrm{~m}$ na linha de nebulização. Os ventiladores utilizados na climatização das instalações eram do tipo axial, tufão, com três pás, motor de $0,5 \mathrm{cv}$, com $91,4 \mathrm{~cm}$ de diâmetro, $1120 \mathrm{rpm}$ de rotação e vazão de $5 \mathrm{~m}^{3} \mathrm{~s}^{-1}$; já os machos do grupo B foram mantidos em baias climatizadas de acordo com o manejo já realizado pela propriedade, feito de forma manual, no qual havia apenas ventiladores acionados em horário predeterminado, segundo o manejo já aplicado na granja, entre 13 e 13 h 30 min.

Os dados do ambiente interno foram registrados por meio de seis transmissores de temperatura e umidade (RHT - NOVUS Produtos Eletrônicos Ltda.), cujos valores medidos são convertidos em sinais de saída de 4 a $20 \mathrm{~mA}$ para a conexão com o datalogger, distribuídos uniformemente entre os dois grupos e conectados a um sistema de aquisição de dados (Fieldlogger - NOVUS Produtos Eletrônicos Ltda.). Já os dados climatológicos externos foram coletados e armazenados por meio de outro equipamento de aquisição de dados (UMMI Betha Eletrônica Ltda.), que possui memória interna para o armazenamento dos dados de temperatura e umidade, tornando possível a avaliação posterior do processo.

A Figura 1 apresenta o desenho esquemático das baias e dos equipamentos empregados no experimento.

Uma análise estatística de correlação e de teste de médias foi realizada entre os dados dos parâmetros ambientais internos e externos à instalação para estabelecer a eficácia da operação do sistema de climatização.

Os índices indicadores das condições microclimáticas foram calculados e analisados com o intuito de se avaliar a adequação do ambiente térmico à zona de conforto predefinida para a categoria dos cachaços no ambiente das instalações de cada um dos grupos e também no ambiente externo ao galpão. Os índices utilizados foram ITU (índice de temperatura e umidade) (Silva, 2000) e Entalpia (Perissinotto, 2002); para o cálculo do ITU, empregou-se a Eq. 1 .

$$
\mathrm{ITU}=\mathrm{Ts}+0,36 \mathrm{Tpo}+41,2
$$

em que:

ITU- índice de temperatura e umidade, adimensional

Ts - temperatura do termômetro de bulbo seco, ${ }^{\circ} \mathrm{C}$

Tpo - temperatura do ponto de orvalho, ${ }^{\circ} \mathrm{C}$

A entalpia foi calculada segundo a Eq. 2.

$$
\mathrm{H}=6,7+0,273 \times \mathrm{Ts}+\frac{\mathrm{UR}}{100 \times 10^{\left(\frac{7,5 \times \mathrm{Ts}}{237,5+\mathrm{Ts}}\right)}}
$$

em que:

$$
\begin{aligned}
& \mathrm{H} \text { - entalpia, } \mathrm{kJ} \mathrm{kg}^{-1} \text { ar seco } \\
& \mathrm{UR} \text { - umidade relativa do ar, \% }
\end{aligned}
$$

Os parâmetros elétricos monitorados foram demanda máxima, fator de potência e consumo de energia elétrica. Os parâmetros relativos ao uso da energia elétrica pelos equipamentos foram avaliados por meio de dois medidores de energia multivariáveis instalados na caixa de distribuição de circuitos. Verificou-se o consumo de energia elétrica dos equipamentos de climatização no mesmo instante em que os dados relativos à temperatura e umidade relativa do ar, foram coletados. Os registros foram realizados a cada $15 \mathrm{~min}, 24 \mathrm{~h}$ por dia.

O custo médio operacional, que é o custo do consumo de energia elétrica do sistema de climatização completo, foi calculado tendo-se por base o consumo mensal de energia elétrica dos meses de novembro e dezembro (meses completos) medido por meio de dois medidores de energia multivariável (RE 6000 e RE 6081 da EMBRASUL) instalados na caixa de distribuição de circuitos e atendendo aos padrões estabelecidos pela ANEEL (Agência Nacional de Energia).

Na propriedade a inseminação artificial ocorreu diariamente, duas vezes ao dia, às 7 e às $17 \mathrm{~h}$. Cada fêmea foi inseminada três vezes por cio, com doses inseminantes de dois a três diferentes cachaços, em virtude da necessidade de um intervalo mínimo entre coletas de sêmen de um mesmo reprodutor e ao grande número de fêmeas em relação ao de machos. O sêmen dos cachaços participantes do experimento foi coletado

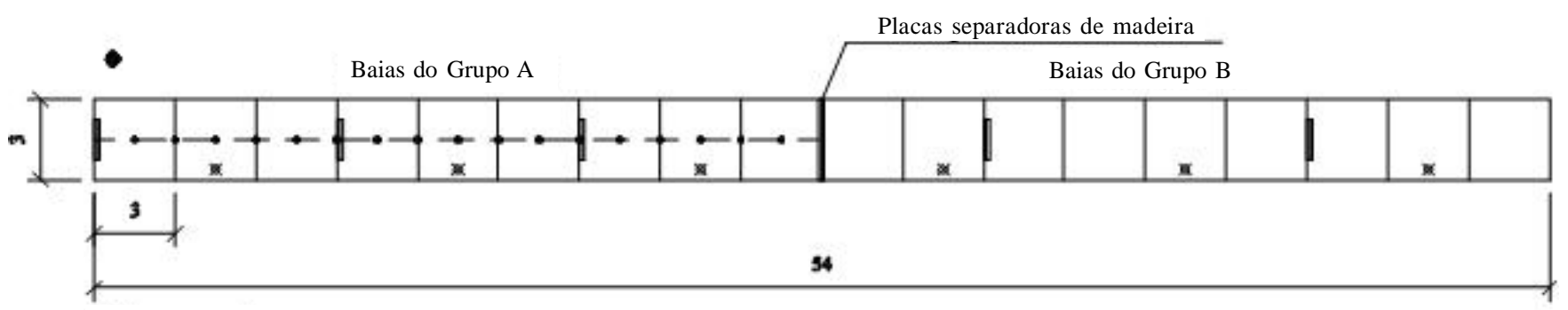

Cotas em metros

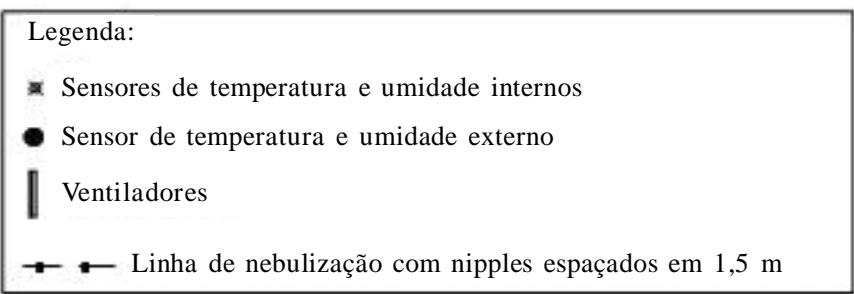

Figura 1. Desenho esquemático da localização dos equipamentos de climatização e medição nos tratamentos 
respeitando-se o manejo reprodutivo já realizado na propriedade com intervalo de uma semana e no período da pesquisa coletaram-se, portanto, 11 amostras.

Os dados reprodutivos foram avaliados de acordo com os valores estipulados pelo manual para exame andrológico e avaliação de sêmen animal (CBRA, 1998). Uma análise minuciosa das características macro e microscópicas das amostras de sêmen foi realizada semanalmente, em laboratório; a avaliação macroscópica consistiu em avaliar características gerais do ejaculado, tais como: volume, cor e aspecto. Por meio da avaliação microscópica analisaram-se características específicas, tais como: motilidade, vigor, aglutinação e concentração espermática, integridade do acrossomo e morfologia espermática. Neste último item foram analisadas tanto as porcentagens de espermatozóides normais e anormais como as de defeitos espermáticos, tais como cabeça solta, defeitos de colo e de cauda (cauda dobrada ou enrolada), gota proximal e defeitos de peça intermediária.

A análise estatística dos dados foi realizada pelo programa computacional SAS (Statistical Analysis System), com nível de significância de $5 \%$. O coeficiente de correlação de Pearson $(-1 \leq \mathrm{r} \leq 1)$ foi utilizado para verificar a correlação entre os dados climáticos internos dos dois grupos avaliados e os dados climáticos externos. Para verificação de diferença estatística entre os dados ambientais dos grupos, utilizaramse os testes de Kolmogorov-Smirnov e o de Wilcoxon. Testes de aderência para a distribuição normal dos dados (ShapiroWilk, Kolmogorov-Smirnov, Cramer-von Mises e AndersonDarling) foram realizados para cada variável relacionada ao sêmen dos cachaços de cada grupo experimental.

\section{Resultados E DisCussão}

O resultado obtido pelo teste de Kolmogorov-Smirnov, que comparou os dados de entalpia e ITU entre os sensores dos grupos A e B e a média dos dados dos grupos em todo o período com os do ambiente externo ao galpão, demonstrou que a diferença entre as médias foi significativa, conforme pode ser constatado na Tabela 1.

Na comparação entre os dados de ITU e entalpia dos grupos A e B também foi encontrada diferença estatística ( $p<0,0001)$, sendo a média de entalpia do grupo A ligeiramente superior à do grupo B; já com relação ao ITU, ocorreu o inverso, ou seja, a média se mostrou mais elevada no grupo B demonstrado que, apesar de ambos os grupos apresentarem um valor médio

Tabela 1. Valores médios* de entalpia e índice de temperatura e umidade (ITU)

\begin{tabular}{|c|c|c|c|c|}
\hline & \multicolumn{2}{|c|}{ Entalpia } & \multirow{2}{*}{$\begin{array}{c}\text { ITU } \\
\text { (adimensional) }\end{array}$} & \multirow{2}{*}{$\begin{array}{c}\text { Erro padrão } \\
\text { ITU }\end{array}$} \\
\hline & $\left.(\mathrm{kJ} \mathrm{kg})^{-1}\right)$ & Erro padrão & & \\
\hline Sensor 1 & 70,38 & 4,15 & 73,23 & 3,20 \\
\hline Sensor 2 & 69,64 & 4,11 & 72,49 & 3,09 \\
\hline Sensor 3 & 69,47 & 3,88 & 72,32 & 2,84 \\
\hline Média do Grupo A & $70,42 \mathrm{a}$ & 4,07 & $72,76 b$ & 3,06 \\
\hline Sensor 4 & 69,73 & 3,84 & 72,68 & 2,87 \\
\hline Sensor 5 & 70,50 & 5,38 & 72,80 & 4,06 \\
\hline Sensor 6 & 72,18 & 5,46 & 73,79 & 4,00 \\
\hline Média do Grupo B & $69,75 \mathrm{~b}$ & 5,07 & 73,09 a & 3,73 \\
\hline Sensor externo & $71,18 \mathrm{c}$ & 8,59 & $72,96 \mathrm{c}$ & 6,49 \\
\hline
\end{tabular}
estatisticamente $(p<0,05)$ de ITU que classifica o ambiente como sendo crítico para os animais, o ambiente do grupo A mostrou-se ligeiramente melhor do que o do grupo B. As médias de entalpia e de ITU, apesar de ultrapassarem os limites de segurança, que são de 60,44 a 68,62 para entalpia e de até 70 para ITU, estão muito próximas a eles, tanto nos grupos A e B quanto no ambiente externo.

O teste utilizado para análise de temperatura foi o de Wilcoxon cuja escolha se baseou no fato dos valores absolutos das médias de temperatura dos grupos A e B e do sensor externo, estarem muito próximos uns dos outros; os valores médios de temperatura e umidade estão expressos na Tabela 2.

Tabela 2. Valores médios* de temperatura e umidade relativa do ar

\begin{tabular}{|c|c|c|c|c|}
\hline & \multicolumn{2}{|c|}{ Temperatura } & \multicolumn{2}{|c|}{ Umidade relativa } \\
\hline & $\left({ }^{\circ} \mathrm{C}\right)$ & Erro padrão & $(\%)$ & Erro padrão \\
\hline Sensor 1 & 24,92 & 2,97 & 64,83 & 11,85 \\
\hline Sensor 2 & 24,10 & 2,79 & 68,76 & 11,40 \\
\hline Sensor 3 & 23,93 & 2,48 & 69,32 & 10,97 \\
\hline Média do Grupo A & 24,32 a & 2,79 & 67,64 a & 11,58 \\
\hline Sensor 4 & 24,38 & 2,56 & 66,44 & 10,42 \\
\hline Sensor 5 & 24,07 & 3,65 & 73,64 & 14,20 \\
\hline Sensor 6 & 24,70 & 3,56 & 75,31 & 13,84 \\
\hline Média do Grupo B & $24,38 \mathrm{a}$ & 3,31 & $71,86 \mathrm{~b}$ & 13,51 \\
\hline Sensor externo & $24,20 \mathrm{~b}$ & 5,97 & $77,26 \mathrm{c}$ & 22,83 \\
\hline
\end{tabular}

A correlação entre os dados de temperatura e umidade relativa dos grupos A e B pode ser considerada estatisticamente significativa porém, de acordo com o teste de Wilcoxon, os valores médios de temperatura entre os grupos $\mathrm{A}$ e $\mathrm{B}$ não diferiram estatisticamente ( $\mathrm{p}$-value $=0,25$ ), visto que o valor do nível de significância estipulado para o teste foi de 0,05 . Os valores médios de umidade relativa dos grupos A e B diferiram, sendo o valor médio de umidade maior no grupo B. Esses resultados diferem dos encontrados por Suriyasomboon et al. (2004) e Kunavongkrit et al. (2005), que encontraram temperaturas máximas inferiores e umidade superior no sistema evaporativo, quando comparado ao convencional.

Verificou-se significativa diferença estatística entre as médias de temperatura de ambos os grupos em relação às medias obtidas no ambiente externo, sendo esta inferior às médias obtidas no ambiente interno. Este resultado também foi encontrado por Sarubbi et al. (2008). Isto se deve às temperaturas mais baixas ocorridas no período da noite e que, juntamente com a expressiva produção de calor pelos animais no interior da instalação, fizeram com que o ambiente externo apresentasse tal resultado, que não reflete a situação ocorrida durante as horas mais quentes do dia; no entanto, também foi no ambiente externo que os maiores índices de perigo e emergência foram encontrados, conforme Tabela 3.

Tabela 3. Frequência de tempo das condições ambientais segundo o índice de temperatura e umidade (ITU)

\begin{tabular}{lcccc}
\hline & $\begin{array}{c}\text { Seguro } \\
\text { (Até 70) }\end{array}$ & $\begin{array}{c}\text { Crítico } \\
\text { (71 a 78) }\end{array}$ & $\begin{array}{c}\text { Perigo } \\
\text { (79 a 83) }\end{array}$ & $\begin{array}{c}\text { Emergência } \\
\text { (Acima de 83) }\end{array}$ \\
\cline { 2 - 5 } & & \multicolumn{3}{c}{ (\%) } \\
Ambiente externo & 33,1 & 47,4 & 11,2 & 8,3 \\
Grupo A & 17,5 & 77,8 & 4,5 & 0,2 \\
Grupo B & 19,2 & 70,0 & 10,4 & 0,4 \\
\hline
\end{tabular}


Com base nos estudos desenvolvidos por Suriyasomboon et al. (2004), que afirmaram que o volume e a produção espermática total diminuem quando a temperatura e a umidade excedem, respectivamente, $27^{\circ} \mathrm{C}$ e $70 \%$ no sistema climatizado, os resultados encontrados demonstraram que no grupo A a temperatura esteve acima do limite máximo de $27^{\circ} \mathrm{C}$ em $13 \%$ do tempo (Figura 2A) e a umidade relativa esteve acima de $70 \%$ em $47,5 \%$ do tempo (Figura $2 \mathrm{~B}$ ) enquanto no grupo B a temperatura excedeu os $27^{\circ} \mathrm{C} \mathrm{em} 18 \%$ do tempo e a umidade ultrapassou os 70 em $58 \%$ do tempo.

De acordo com a classificação de ITU (Silva, 2000), tanto no grupo A quanto no grupo B, na maior parte do tempo os valores de ITU expressaram uma condição ambiental considerada crítica para os suínos; no entanto, o grupo A apresentou porcentagens de índices de perigo e emergência consideravelmente menores que o grupo B.

Para os parâmetros seminais avaliados a Tabela 4 apresenta os resultados encontrados.

Dentre os parâmetros seminais avaliados pode-se considerar que apenas as médias das variáveis referentes ao volume seminal e a concentração espermática diferiram estatisticamente, sendo as médias do grupo B superiores às do grupo A. Esses resultados são semelhantes aos relatados por Suriyasomboon et al. (2004), que não encontraram efeito significativo do sistema de climatização evaporativo sobre o volume do ejaculado e a produção espermática total. As demais variáveis, embora tenham apresentado diferença em números absolutos, não demonstraram diferença estatística significativa.

A.

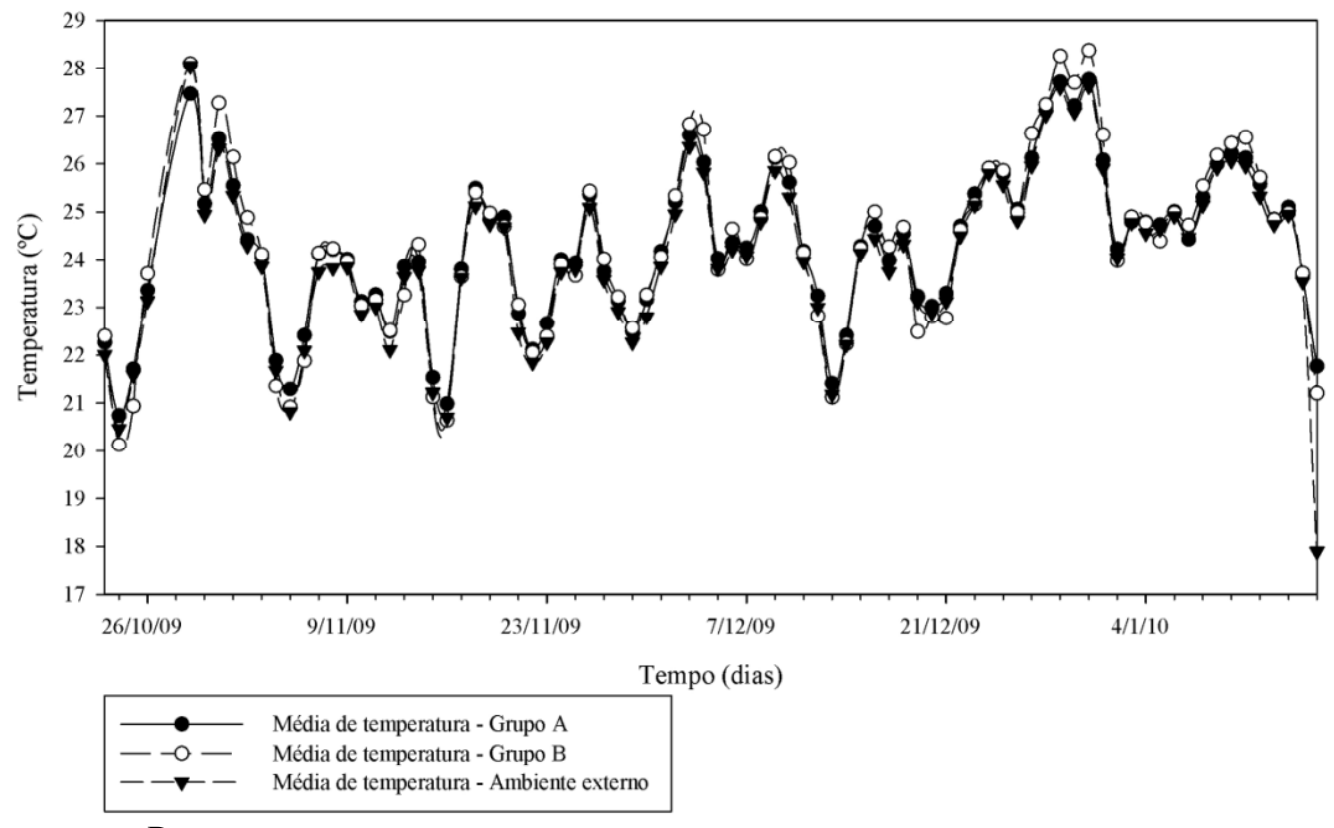

B.

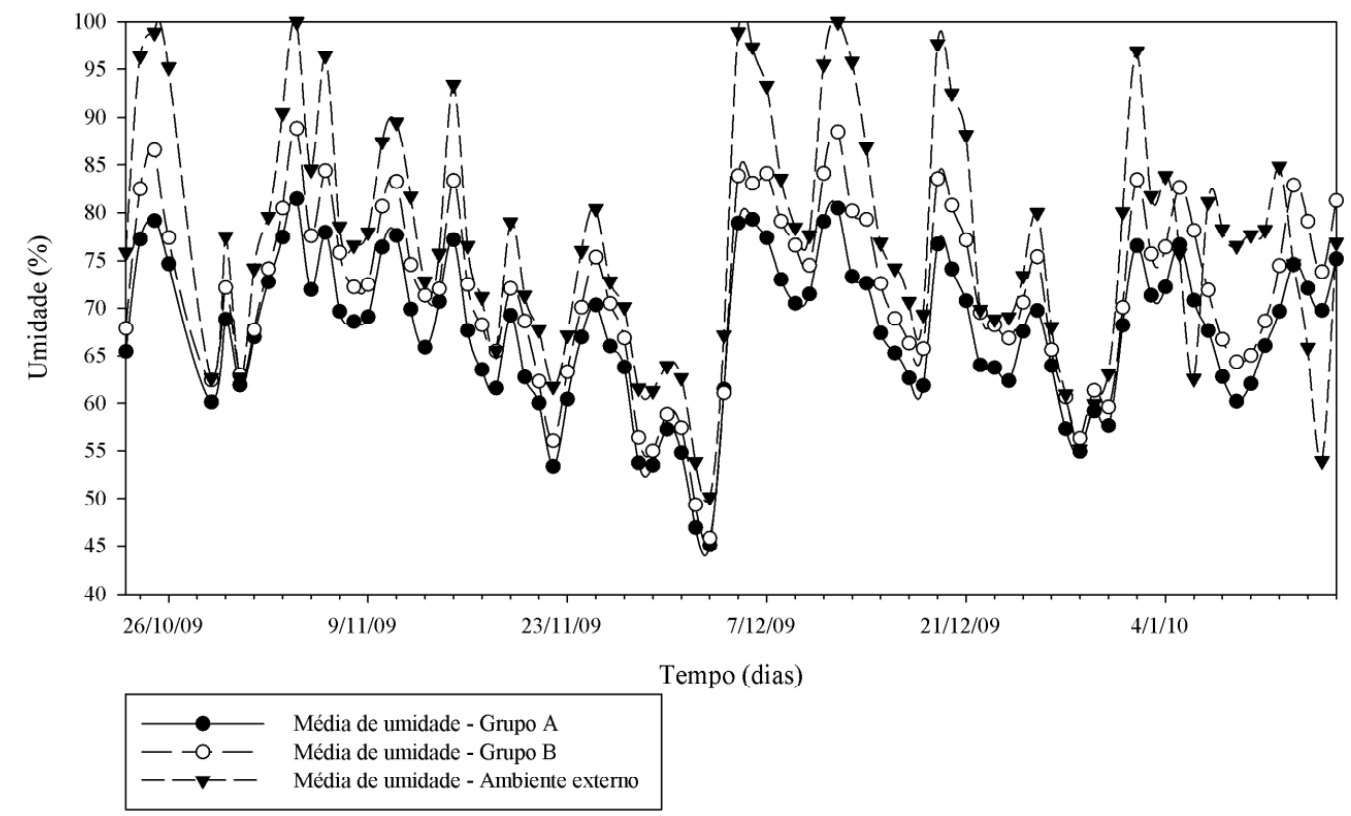

Figura 2. Comparação entre as médias diárias de temperatura (A) e comparação entre as médias diárias de umidade relativa (B) 
Tabela 4. Médias* das variáveis reprodutivas

\begin{tabular}{|c|c|c|c|c|}
\hline & \multicolumn{2}{|c|}{ Grupo A } & \multicolumn{2}{|c|}{ Grupo B } \\
\hline & Média & $\mathrm{EP}^{* *}$ & Média & EP $^{* *}$ \\
\hline Volume $(\mathrm{mL})$ & 259,66 a & 80,69 & $294,75 b$ & 67,75 \\
\hline Motilidade (\%) & $88,44 a$ & 7,37 & $88,62 \mathrm{a}$ & 4,68 \\
\hline Concentração (bilhões) & $92,96 \mathrm{a}$ & 20,86 & $107,96 \mathrm{~b}$ & 21,65 \\
\hline$\%$ de espermatozóides normais & $98,44 a$ & 2,45 & 98,62 a & 2,24 \\
\hline$\%$ de espermatozóides anormais & $1,55 \mathrm{a}$ & 2,45 & $1,37 \mathrm{a}$ & 2,25 \\
\hline Acrossoma (\%) & $0,63 a$ & 0,58 & $0,55 a$ & 0,52 \\
\hline Cabeça solta (\%) & $0,30 \mathrm{a}$ & 0,48 & $0,21 a$ & 0,43 \\
\hline Colo (\%) & $0,47 a$ & 0,65 & $0,34 a$ & 0,52 \\
\hline Defeito de cauda (\%) & $0,53 a$ & 1,06 & $0,50 \mathrm{a}$ & 1,08 \\
\hline Cauda dobrada & $1,52 \mathrm{a}$ & 1,26 & $1,44 a$ & 1,39 \\
\hline Cauda enrolada & $0,11 \mathrm{a}$ & 0,31 & $0,11 \mathrm{a}$ & 0,31 \\
\hline Gota protoplasmática proximal (\%) & $0,65 a$ & 0,62 & $0,67 a$ & 0,53 \\
\hline Peça intermediária (\%) & $0,99 a$ & 0,70 & $0,79 a$ & 0,67 \\
\hline
\end{tabular}

* valores de médias seguidos de letras diferentes na linha diferem estatisticamente $(p<0,05)$ ** EP - erro padrão

Avaliando os parâmetros de consumo do sistema de climatização completo contidos na Tabela 5, observa-se que os ventiladores e a bomba de nebulização apresentaram consumo de energia elétrica bastante variável.

Tabela 5. Valores do consumo de energia elétrica do sistema de climatização completo

\begin{tabular}{lccr}
\hline \multirow{1}{*}{ Mês } & $\begin{array}{c}\text { Consumo/ } \\
\text { Ventiladores }\end{array}$ & $\begin{array}{c}\text { Consumo/Bomba de } \\
\text { nebulização }\end{array}$ & \multicolumn{1}{c}{ Total } \\
\cline { 2 - 3 } & \multicolumn{3}{c}{$\mathbf{( k W h )}$} \\
Outubro & 20,054 & 20,563 & 40,617 \\
Novembro & 279,339 & 85,553 & 364,492 \\
Dezembro & 411,695 & 133,076 & 544,771 \\
Janeiro & 216,462 & 51,038 & 267,500 \\
Total & 927,550 & 290,23 & $1.217,780$ \\
\hline
\end{tabular}

Tal fato se deve, sobremaneira, ao acionamento automático que, por sua vez, era controlado pelas condições de temperatura e umidade relativa no interior da instalação. Comparando-se o consumo de energia elétrica dos ventiladores com o da bomba de nebulização, constata-se que os níveis de consumo diário dos ventiladores são bastante superiores aos da bomba, devido ao fato dos ventiladores se encontrarem em maior número, além de funcionarem por um intervalo de tempo maior.

O custo total de investimento no sistema de climatização foi $\mathrm{R} \$ 3.306,80$, que corresponde à soma dos custos de aquisição do sistema hidráulico, do sistema de ventilação e do controlador automático.

Os custos médios operacionais, que constituem o custo do consumo de energia elétrica do sistema de climatização completo, foram calculados tendo-se por base o consumo mensal de energia elétrica dos meses de novembro e dezembro (meses completos) e o valor da tarifa de consumo de energia elétrica da concessionária que opera na cidade, que era de $\mathrm{R} \$ 0,19298$ por $\mathrm{kWh}$; como o mês de novembro apresentou um consumo de $364,492 \mathrm{kWh}$, seu custo médio foi de R $\$ 70,34$ e, para o mês de dezembro foi encontrado um consumo de 544,771 $\mathrm{kWh}$ com custo médio de $\mathrm{R} \$ 105,13$.

De acordo com a empresa, no transformador que abastece a instalação em análise, as contas de energia elétrica referentes aos meses de novembro e dezembro de 2009 foram, respecti- vamente, de $\mathrm{R} \$ 3.306,66$ e R\$2.181,16. Portanto, o impacto do custo médio nesses mesmos meses seria, respectivamente, de 2,26 e de $4,82 \%$, o qual pode ser considerado baixo.

\section{ConClusões}

1. O emprego da climatização automatizada não produziu efeito significativo no sistema de climatização evaporativo (Grupo A); além disto, não houve melhoria nos índices de qualidade seminal dos animais deste grupo.

2. Os valores médios de volume seminal e a concentração espermática foram estatisticamente superiores nos animais do grupo B.

3. A nebulização não foi capaz de reduzir sensivelmente a média de temperatura do grupo A; apesar disto, o ambiente interno do grupo A esteve em maior conformidade com os padrões de conforto térmico dos animais.

4. O incremento ocasionado pelo uso dos equipamentos de climatização e a automação, não representam um fator limitante na instalação nem na utilização do sistema, pois não acarretará acréscimo significativo na conta de energia elétrica do galpão.

\section{LITERATURA CITADA}

Albright, L. D. Environment control for animals and plants. St. Joseph: American Society of Agricultural Engineers, 1990. 453p.

Bortolozzo, F. P.; Dallanora, D.; Bernardi, M. L.; Bennemann, P. E.; Wentz, I. Técnicas associadas à inseminação artificial no suíno que visam a redução do número de espermatozóides necessários por fêmea ao ano. Revista Brasileira de Reprodução Animal, v.27, p.133-139, 2003.

Bueno, L. G. Rossi, L. A. Comparação entre tecnologias de climatização para criação de frangos quanto a energia, ambiência e produtividade. Revista Brasileira de Engenharia Agrícola eAmbiental, v.10, p.497-504, 2006.

Cavalcanti, S. S. Suinocultura dinâmica. Belo Horizonte: Itapuã Editora e Gráfica Ltda, 1998. 494p.

CBRA - Colégio Brasileiro de Reprodução Animal. Manual para exame andrológico e avaliação de sêmen animal. Belo Horizonte: Colégio Brasileiro de Reprodução Animal, 2.ed., 1998. 49p.

Curtis, S. E. Environmental management in animal agriculture. Ames: Iowa State University Press, 1983. p.97-122.

Estienne, M. J. Keep boars cool during summer. Livestock Update. Virginia Cooperative Extension. 2000. <http:// www.ext.vt.edu/news/periodicals/livestock/aps-00_07/aps0246.html>. 9 Mai. 2006.

Huynh, T. T. T.; Aarnink, A. J. A.; Spoolder, H. A. M.; Verstegen, M. W. A.; Kemp, B. Effects of floor cooling during high ambient temperatures on the lying behavior and productivity of growing finishing pigs. Transactions of the ASAE, v.47, p.1773-1782, 2004. 
Kunavongkrit, A.; Suriyasomboon, A.; Lundheim, N.; Heard, T.; Einarsson, S. Management and sperm production of boars under differing environmental conditions. Theriogenology, v.63, p.657-667. 2005.

Pedersen, S. Heat and moisture production for pigs on animal and house level. ASAE Meeting Presentatiom. Paper n. 024178. 2002.

Perissinotto, M. Avaliação da eficiência produtiva e energética de sistemas de climatização em galpões tipo "freestall" para confinamento de gado leiteiro. Piracicaba: USP, 2002. 122p. Dissertação Mestrado

Sarubbi, J.; Rossi, L. A.; Laranjeira, E. G.; Oliveira, R. A.; Velloso, N. M. Power-saving procedures and animal thermal comfort at a growing/finishing swine production unit. Revista Brasileira de Engenharia de Biossistemas, v.2, p.185-192, 2008.
Sarubbi, J.; Rossi, L. A. ; Moura, D. J.; Oliveira, R. A.; David, E. Utilização de energia elétrica em diferentes sistemas de aquecimento para leitões desmamados. Engenharia Agrícola, v.30, p.1003-1011, 2010.

Silva, R. G. Introdução à bioclimatologia animal. São Paulo: Nobel, 2000. 286p.

Suriyasomboon, A. Herd investigations on sperm production in boars, and sow fertility under tropical conditions. Uppsala: Swedish University of Agricultural Sciences. 2005. 52p. Tese Doutorado

Suriyasomboon, A.; Lundeheim, N.; Kunavongkrit, A.; Einarsson, S. Effect of temperature and humidity on sperm production in Duroc boars under different housing systems in Thailand. Livestock Production Science, v.89, p.19-31. 2004.

Tolon, Y. B.; Baracho, M. S.; Nããs, I. A.; Rojas, M.; Moura, D. J. Ambiências térmica, aérea e acústica para reprodutores suínos. Engenharia Agrícola, v.30, p.1-13, 2010. 